\title{
Controlador MPPT baseado em Lógica Fuzzy Aplicado a um Sistema Fotovoltaico Conectado à uma Rede
}

\author{
Arthur Caio Vargas e Pinto* Tiago Henrique Santa Maria** \\ Janaina Gonçalves Oliveira ${ }^{* *}$ Andre Luis Marques Marcato** \\ Eduardo Pestana de Aguiar** \\ * Departamento de Eletroeletrônica, Centro Federal de Educação \\ Tecnológica de Minas Gerais (CEFET-MG),(arthuki@gmail.com) \\ ** Programa de Pós Graduação em Engenharia Elétrica, Universidade \\ Federal de Juiz de Fora, $M G$, \\ (tiago.santamaria@engenharia.ufjf.br)(janaina.oliveira@ufjf.edu.br) \\ (andre.marcato@ufjf.edu.br)(eduardo.aguiar@engenharia.ufjf.br)
}

\begin{abstract}
Among the renewable energies sources, the solar have excelled from the others because its fast growning and global accept. But, photovoltaic systems have low efficiency and the cause is related with various variables, as temperature, irradiation, angle of the panels, and others. The implementation and operation of photovoltaic cells requires few controls one of them is, tracking the point of maximum potency of the panel (MPPT). This paper proposes an MPPT algorithm based on Fuzzy Logic. Fuzzy algorithms has its advantages like fast responses not dependent on variations in the system and no mathematical equations involved. The following simulations show us the fuzzy controller works efficiently. The proposed scheme was made using Matlab/Simulink.

Resumo: Entre as fontes de energia renováveis existentes, a energia solar fotovoltaica tem se destacado pelo seu rápido crescimento e aceitação mundial. Entretanto, sistemas fotovoltaicos possuem baixa eficiência e seu rendimento está relacionado a vários fatores, como temperatura, irradiação, angulação dos painéis, entre outros. Assim, a implementação e operação eficiente de geradores fotovoltaicos requerem a utilização de um sistema Seguidor de Máxima Transferência de Potência - MPPT. Este trabalho propõe o desenvolvimento de um algoritmo MPPT baseado em lógica fuzzy, capaz de fazer com que o sistema opere no ponto de máxima potência mesmo sob condições variadas de trabalho, aumentando a eficiência. Um algoritmo fuzzy tem como vantagem a rápida resposta à variações nas condições do sistema e a não necessidade de modelagem matemática do sistema, como ocorre em outros métodos. Os resultados das simulações indicam que o controlador fuzzy opera de forma eficiente na manutenção da geração da máxima potência pelo sistema. O sistema proposto foi criado e analisado utilizando a ferramenta Matlab/Simulink.
\end{abstract}

Keywords: Photovoltaic Systems; Fuzzy Logic; Power Eletronics; MPPT; Control

Palavras-chaves: Sistemas fotovoltaicos; Lógica fuzzy; Inteligência computacional; MPPT; Eletrônica de Potência; Controle

\section{INTRODUÇÃO}

Tendo em vista a crescente demanda mundial de energia, o uso de combustíveis fósseis tem sido muito debatido devido à poluição e outros impactos ambientais causados pelo consumo de energia não renovável. O efeito estufa e o aquecimento global, conseqüências da queima de combustíveis fósseis, têm um sério impacto na vida, devido às significativas mudanças climáticas observadas nos últimos anos. Além disso, há um consenso de que a disponibilidade de combustíveis fósseis para uso de energia tende a diminuir, o que compromete a segurança e autonomia energética global. Esse cenário aumentou a conscientização ambiental dos países em busca de fontes alternativas de energia capazes de suprir sua demanda energética de forma sustentável.

Entre as fontes de energia renováveis existentes, a energia solar fotovoltaica tem se destacado pelo seu rápido crescimento e aceitação mundial, uma vez que atualmente representa uma parte significativa da matriz energética de alguns países. Entretanto, sistemas fotovoltaicos possuem baixa eficiência e seu rendimento está relacionado a vários fatores, como temperatura, irradiação, angulação dos painéis, entre outros. Estando submetidos às variações climáticas ao longo do dia, a implementação e operação eficiente de geradores fotovoltaicos requerem a utilização de um sistema Seguidor de Máxima Transferência de Potência 


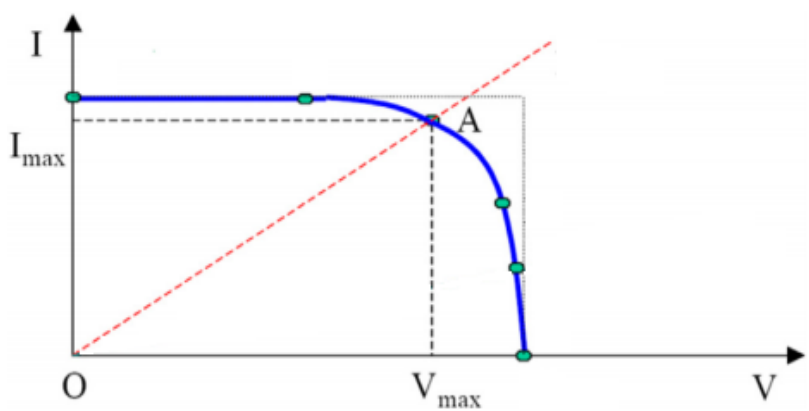

Figura 1. Curva característica de uma célula fotovoltaica. Fonte: Adaptado de Khatib et al. (2018)

(do inglês, Maximum Power Point Tracking - MPPT), a fim de se conseguir produzir a máxima potência possível.

A característica elétrica de uma célula é geralmente representada pela sua curva de corrente versus tensão (IV), denominada curva característica. Toda célula possui uma curva característica IV, que é capaz de descrever o comportamento desta célula em diferentes situações. Durante seu funcionamento e de acordo com a carga ligada ao sistema, a irradiação solar e a temperatura dos painéis, a célula tem um ponto de trabalho situado sobre esta curva, dependendo das condições a que está submetida. A Figura 1 mostra um exemplo de curva característica.

Algortimos de MPPT utilizam a tensão e a corrente geradas pelo sistema fotovoltaico para regular o ciclo de trabalho (do inglês, duty cycle) do sinal de Pulse Width Modulation - PWM aplicado no chaveamento do conversor $\mathrm{CC} / \mathrm{CC}$ presente no sistema, consequentemente fazendo com que o sistema opere no ponto de máxima transferência de potência - PMP (Hashim et al., 2018) (representado pelo ponto "A" na Figura 1). Atualmente existem diferentes metodologias de implementação de algoritmos MPPT, destacando-se a Perturbe e Observe ( $\mathrm{P} \& \mathrm{O})$ e a Condutância Incremental, que são algoritmos que se baseiam nas medições de tensão e corrente dos painéis para realizar a tomada de decisão sobre o aumento ou diminuição da variável sendo controlada (Soulatiantork et al., 2018). O algoritmo Perturbe e Observe (P \& O) é o mais utilizado na prática devido à sua facilidade de implementação e consiste em perturbar a tensão do sistema em uma direção e obeservar a potência gerada. Quando a variação de potência é positiva, o ponto de operação foi movido para uma posição mais próxima ao PMP, dessa forma a perturbação continua na mesma direção. Caso contrário, o sentido é invertido. Como este processo é repetido continuamente, o PMP nunca é atingido, ficando o sistema oscilando próximo a este. Abdel-Salam et al. (2018) investigam a fundo o desempenho do método $(\mathrm{P} \& \mathrm{O})$ para rastreamento do PMP sob diferentes condições de funcionamento do sistema fotovoltaico, chegando à conclusão de que este método não é muito eficiente quando os níveis de irradiação variam constantemente. Por isso, desenvolvem um método ( $\mathrm{P} \& \mathrm{O})$ otimizado capaz de reagir melhor a estas oscilações. Já Karabacak (2019) utiliza o método (P \& O) para realizar o MPPT em um sistema eólico, propondo uma melhoria e criando um novo algoritmo que utiliza a inércia da turbina como parâmetro para a determinação do duty cycle do sistema. Por sua vez, o método de Condutância

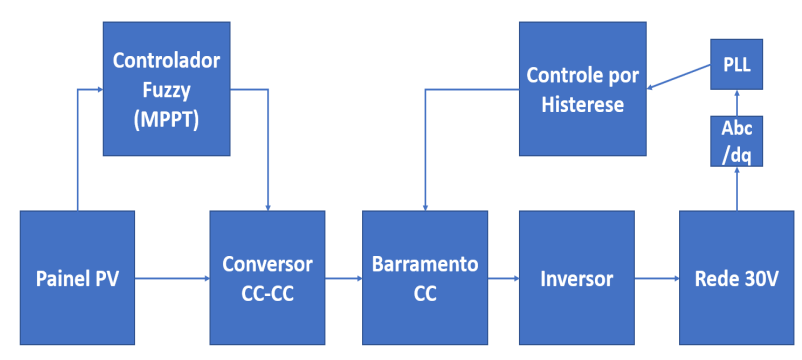

Figura 2. Diagrama geral do sistema ligado a uma rede de 30 Volts.

Incremental utiliza as medidas de corrente e tensão para derivar os valores da condutância instatânea e incremental do sistema, podendo-se então descobrir em que posição da curva característica o sistema está operando. Este conceito é explorado no trabalho de (Kumar et al., 2018), onde observa-se a construção de um algortimo MPPT autoadaptativo, que apresenta pouca oscilação e velocidade resposta superior aos métodos tradicionais de Condutâncial Incremental.

Lógica fuzzy também pode ser utilizada para a criação de algoritmos de rastreamento MPPT, possuindo a vantagem de que o método MPPT usando lógica fuzzy utiliza apenas os valores de corrente e tensão geradas pelo painel, não sendo necessárias informações a respeito dos parâmetros do sistema, como ocorre com outras metodologias. Além disso, algoritmos MPPT fuzzy funcionam sem a necessidade de se realizar cálculos e modelagens matemáticas complexas. O princípio de funcionamento do algoritmo fuzzy MPPT se baseia na análise do ponto de operação do sistema de acordo com sua curva característica. Quando projetado corretamente, um sistema MPPT fuzzy é capaz de superar controladores mais complexos porque fornece uma saída precisa mesmo sem o uso de observadores, como mostra Farajdadian e Hosseini (2019) ao comparar os resultados obitidos usando lógica Fuzzy com o funcionamento do método Perturbe e Observe na criação de um algoritmo MPPT de um sistema fotovoltaico ligado à rede. Por sua vez, Yilmaz et al. (2018) utilizaram lógica fuzzy em um sistema fotovoltaico isolado para otimizar o carregamento de baterias, alcançando até $5 \%$ de melhora na precisão do carregamento, reduzindo perdas e aumentando a vida útil das baterias. Neste trabalho os resultados foram otimistas e a lógica fuzzy criada foi capaz de encontrar o ponto de máxima potência sem perder a efetividade do painel solar, e manteve-se com uma resposta rápida quanto às variações impostas na entrada do sistema. Este artigo fornece uma nova abordagem ao utilizar o fuzzy MPPT em um sistema fotovoltaico conectado a uma micro-rede simulada, trazendo a possibilidade de posterior implementação do modelo em uma planta real.

O sistema fotovoltaico proposto é composto por painéis solares, um conversor CC-CC, controlador fuzzy MPPT, um inversor trifásico e o equipamento de conexão com a rede. O esquemático do sistema é mostrado na Figura 2. 


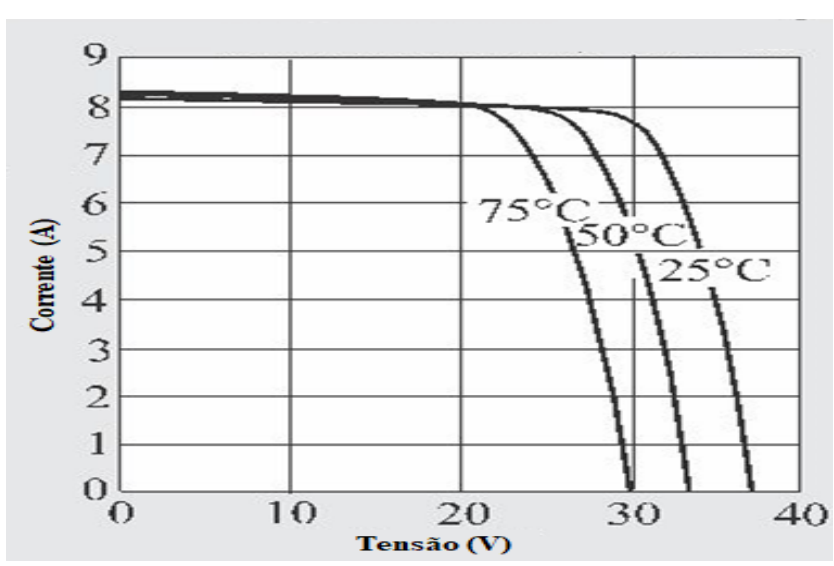

Figura 3. Comportamento da curva característica de uma célula solar de acordo com a variação de temperatura, para $1000 \mathrm{~W} / \mathrm{m}^{2}$.

Fonte: Coelho e Martins (2012)

\section{O CONCEITO DE PONTO DE MÁXIMA TRANSFERÊNCIA DE POTÊNCIA}

Toda célula possui uma curva característica IV, que é capaz de descrever o comportamento desta célula em diferentes situações. Durante seu funcionamento, a célula tem um ponto de trabalho situado sobre esta curva, dependendo das condições a que está submetida.

Com relação à curva característica de células, é importante entender os seguintes conceitos:

(1) Corrente de curto circuito $\left(\boldsymbol{I}_{S C}\right)$ : Corrente máxima produzida pela célula solar, a uma tensão igual a zero.

(2) Tensão de circuito aberto $\left(\boldsymbol{V}_{\boldsymbol{O}}\right)$ : Tensão máxima do dispositivo, a uma corrente igual a zero.

(3) Potência máxima $\left(\boldsymbol{P}_{\boldsymbol{M A X}}\right)$ : Potência máxima produzida pelo dispositivo, correspondente ao par máximo $I \times{ }^{x} V$

(4) Corrente no ponto de máxima potência $\left(\boldsymbol{I}_{M A X}\right)$ : É o valor da corrente para $P_{M A X}$.

(5) Tensão no ponto de máxima potência $\left(V_{M A X}\right)$ : É o valor de tensão para $P_{M A X}$.

Uma célula solar produz a máxima quantidade de energia quando opera no ponto de máxima potência, ou seja, no joelho da curva I-V. Variações na temperatura da célula, radiação incidente ou mesmo alteração da carga fazem com que a curva $\mathrm{I}-\mathrm{V}$ se altere, alterando também o ponto de operação e consequentemente a geração de energia elétrica. A corrente gerada pela célula é afetada diretamente pela radiação solar incidente, enquanto que variações na temperatura afetam principalmente a tensão na célula. A tensão na célula é inversamente proporcional à temperatura do dispositivo. $\mathrm{O}$ aumento da temperatura da célula solar fotovoltaica ocasiona uma queda na tensão gerada, consequentemente diminuindo a energia elétrica gerada (Coelho e Martins, 2012). A Figura 3 ilustra o comportamento da curva característica de acordo com a variação de temperatura, a uma irradiação solar constante.

Por outro lado, na Figura 4 observa-se como o aumento da radiação solar incidente ocasiona um aumento na corrente gerada, a uma temperatura constante de 25 graus,

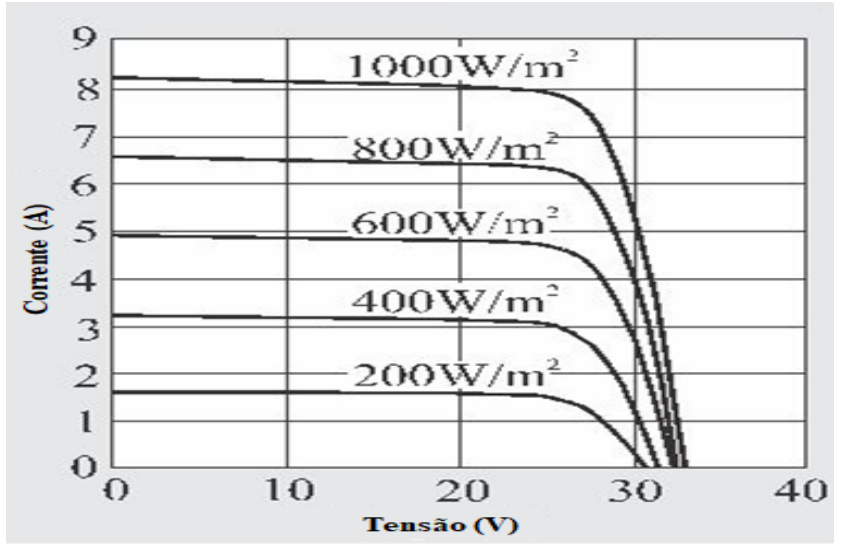

Figura 4. Comportamento da curva característica de uma célula solar de acordo com a variação de irradiação, para $25^{\circ} \mathrm{C}$.

Fonte: Coelho e Martins (2012)

consequentemente aumentando a energia produzida pela célula.

\section{ESTRUTURA DO MODELO}

Um sistema fotovoltaico é composto por uma junção de painéis fotovoltaicos e outros componentes eletrônicos capazes de fazer a conexão do conjunto à baterias, uma carga isolada ou à rede de energia elétrica. Podem variar desde poucos $\mathrm{kW}_{\mathrm{P}}$ a vários $\mathrm{MW}_{\mathrm{P}}$ de potência instalada. Tais sistemas normalmente são compostos por painéis solares, um conversor CC/CC, controlador MPPT, um inversor trifásico e o equipamento de conexão com a rede. O sistema proposto consiste de um único painel de $84 \mathrm{~W}$, conectado a um conversor $\mathrm{CC} / \mathrm{CC}$ boost, por sua vez ligado a um inversor trifásico que alimenta uma minirede de tensão de linha igual a 100V. Foi utilizado um sinal representando a irradiação incidente, assumindo os valores de $1000 \mathrm{~W} / \mathrm{m}^{2}$ e $500 \mathrm{~W} / \mathrm{m}^{2}$, a uma temperatura inicial de $25^{\circ} \mathrm{C}$, mas que sobe para $50^{\circ} \mathrm{C}$.

Os componentes do sistema foram modelados na ferramenta Matlab/Simulink, sobretudo devido à possibilidade de integração entre o sistema fuzzy com o modelo simulado, todos no mesmo ambiente computacional.

\subsection{O sistema fotovoltaico}

Foi utlizado um único painel fotovoltaico, de especificações exibidas na Tabela 1

Tabela 1. Especificações do painel fotovoltaico.

\begin{tabular}{ccc} 
Parâmetro & Variável & Valor \\
\hline Potência máxima & $\mathrm{P}_{\mathrm{MAX}}$ & $84 \mathrm{~W}$ \\
Tensão no PMP & $\mathrm{V}_{\mathrm{MAX}}$ & $17,5 \mathrm{~V}$ \\
Corrente no PMP & $\mathrm{I}_{\mathrm{MAX}}$ & $4,8 \mathrm{~A}$ \\
Tensão de circuito aberto & $\mathrm{V}_{\mathrm{OC}}$ & $24 \mathrm{~V}$ \\
Corrente de curto-circuito & $\mathrm{I}_{\mathrm{SC}}$ & $5,1 \mathrm{~A}$ \\
\hline
\end{tabular}

\subsection{Conversor $C C / C C$ boost}

Conversores boost são também chamados de elevadores de tensão, uma vez que a tensão de saída do conversor é 
superior à sua tensão de entrada. Um conversor boost é composto por um capacitor, um transistor e um diodo e um indutor, que é o mecanismo de armazenamento de energia do conversor. O transistor utilizado é um transistor MOSFET, que é um dispositivo semicondutor. Os parâmetros do conversor boost utilizado encontram-se descritos na Tabela 2.

\section{Tabela 2. Especificações do conversor $\mathrm{CC} / \mathrm{CC}$ boost.}

\begin{tabular}{cc} 
Parâmetro & Valor \\
\hline Indutor $(\mathrm{H})$ & $1.5 \mathrm{e}^{-3}$ \\
Capacitor $(\mu F)$ noPMP & 600 \\
Frequência $(\mathrm{Hz})$ & 15000 \\
Ripple $(\mathrm{A})$ & 0,5 \\
\hline
\end{tabular}

Para dimensionamento do conversor boost foi feito seguindo o padrão adotado no trabalho de Yilmaz et al. (2018), onde a Equação 1 é usada para encontrar o valor referente ao ciclo de trabalho (duty cycle) do conversor.

$$
V_{\text {in }}=V_{\text {out }} \cdot(1-D)
$$

E para dimensionamento do indutor, capacitor e ripple de tensão as Equações 2, 3 e 4 são utilizadas.

$$
\Delta I_{l}=\frac{V_{\text {inmin }} \cdot D}{f s \cdot L}
$$

Sendo $V_{i n(\min )}=$ tensão mínima de entrada, $f_{s}=$ frequencia de chaveamento, $D=$ ciclo de trabalho, $L=$ indutância.

$$
L=\frac{V_{\text {in }} \cdot\left(V_{\text {out }}-V_{\text {in }}\right)}{\Delta I_{l} \cdot f s \cdot V_{\text {out }}}
$$

$V_{\text {out }}=$ Tensão de saída, $V_{\text {in }}=$ Tensão de Entrada, $\Delta I_{l}=$ Ripple de corrente do Indutor (Estimado).

$$
C=\frac{I_{\text {out }} \cdot D}{f s \cdot \Delta V_{\text {out }}}
$$

$C=$ capacitor, $I_{\text {out }}=$ Corrente de saída, $D=$ ciclo de trabalho, $f s=$ frequencia de chaveamento, $\Delta V_{\text {out }}=$ Ripple de tensão de saída.

\subsection{O inversor}

Os inversores são responsáveis para a transformação da Tensão em Corrente contínua em corrente alternada. Com cargas de linha indutivas e resistivas no valor de $1,5 \mathrm{mH} \mathrm{e}$ 0,3 Ohms respectivamente.

Controle por Histerese O controle por histerese é caracterizado pela sua estabilidade incondicionada, resposta rápida e boa precisão. Porém, as técnicas básicas de histerese apresentam também vários aspectos indesejáveis, como frequência de chaveamento variável e alta interferência nas fases no caso de sistemas trifásicos (Buso et al., 2000).

Ganhos do PLL Para os ganhos do PLL foram utilizadas as equações encontadas em Yazdani e Iravani (2010). Um controle mais preciso do PLL mostrou-se útil junto ao controlador de histerese eliminando boa parte do sobresinal dos resultados e um tempo de resposta mais rápido. Um esquema de sistema PLL é mostrado na Figura 5, onde ocorre a conversão do sistema trifásico abc para dq (linear) para ajuste dos ganhos do compensador $\mathrm{H}(\mathrm{s})$, e envialos ao controle por histerese para atuação na tensão do barramento $\mathrm{CC}$.

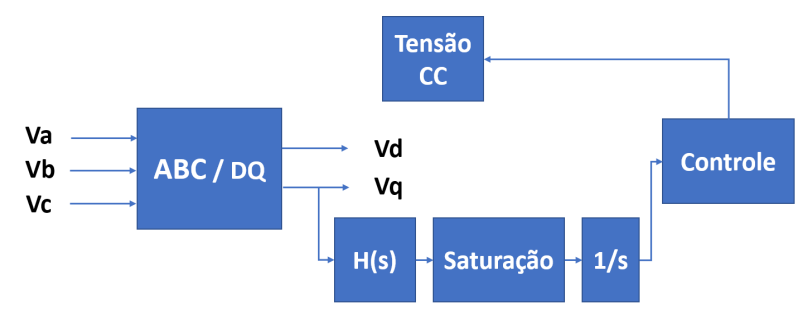

Figura 5. Esquemático utilizado para controle do PLL no inversor.

Fonte: Adaptado de Yazdani e Iravani (2010)

\section{CONTROLADOR FUZZY}

Lógica fuzzy se caracteriza por sua habilidade em imitar o pensamento humano, sendo capaz de traduzir em termos matemáticos a informação imprecisa expressa por um conjunto de regras linguísticas. Se um operador humano for capaz de articular sua estratégia de ação como um conjunto de regras da forma se ... então, um algoritmo passível de ser implementado em computador pode ser construído. O resultado é um sistema de inferência baseado em regras, capaz de fornecer uma sáida com base em parâmetros de entrada.

A maior vantagem de se realizar o controle MPPT utilizando um controlador fuzzy é que não há necessidade de modelar o sistema, como ocorre no caso do projeto de controladores PID, por exemplo. Um sistema MPPT fuzzy funciona com duas entradas: o erro do sistema, representado por E e a variação deste erro, representada por CE. A Equação 1 define estas variáveis. A saída do sistema fuzzy é o ciclo de trabalho (D) que deve ser aplicado ao sinal PWM do transistor do conversor boost.

$$
\begin{gathered}
E(k)=\frac{\Delta P}{\Delta V}=\frac{P(k)-P(k-1)}{V(k)-V(k-1)} \\
C E(k)=E(k)-E(k-1)
\end{gathered}
$$

em que $P k$ e $V k$ são a potência e a tensão geradas pelos painéis, respectivamente, e $P(k-1)$ e $V(k-1)$ são a potência e tensão anteriores geradas pelas sistema fotovoltaico, respectivamente. A lógica fuzzy para a realização do MPPT se baseia no fato de que o controlador deve trabalhar para corrigir o ponto de operação do sistema, aproximando-o ao máximo do ponto de máxima transferência de potência. Obervando a Figura 6, é possível perceber que existem quatro possíveis situações para o ponto de operação, de acordo com as variações na potência e na tensão.

Quando a variação na potência e na tensão é positiva $(P(k)$ $>P(k-1)$ e $V(k)>V(k-1))$, deve haver um incremento na tensão para mover o ponto de operação em direção ao PMP. Caso a variação na potência seja positiva e na tensão seja negativa, a tensão deve ser decrementada. As condições de cada ponto de operação estão detalhadas na Tabela 3.

\subsection{Fuzzyficação}

Em sistemas de Inferência fuzzy, consideram-se entradas não-fuzzy, ou precisas, resultantes de medições ou observações (conjuntos de dados, por exemplo), que é o caso 


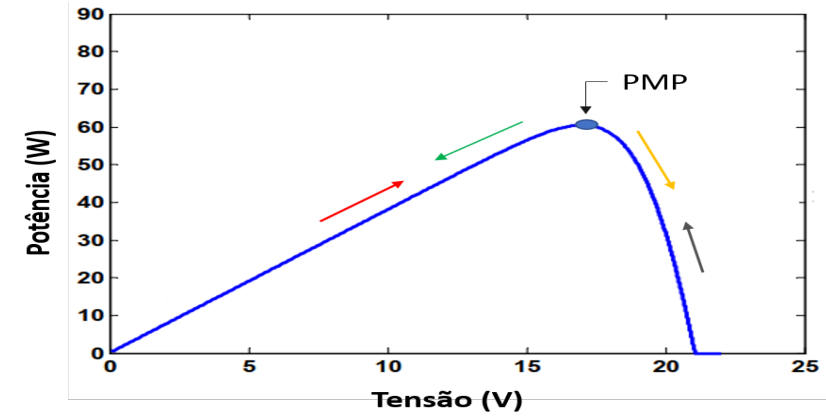

Figura 6. Exemplificação de um ponto de máxima potência.

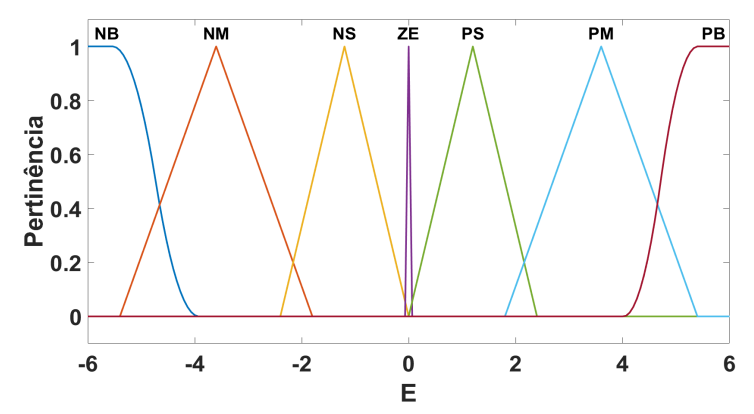

Figura 7. Conjuntos fuzzy relacionados para a variável Erro (E).

da grande maioria das aplicações práticas. Em virtude disto, é necessário efetuar-se um mapeamento destes dados precisos para os conjuntos fuzzy (de entrada) relevantes, o que é realizado no estágio de fuzzyficação. Neste estágio ocorre também a ativação das regras relevantes para uma dada situação.

Foram definidos sete conjuntos fuzzy para representar o erro E e a variação no erro CE: NB (grande negativo), NM (médio negativo), NS (pequeno negativo), ZE (erro zero), PS (pequeno positivo), PM (médio positivo), PB (grande positivo). Para a variável do ciclo de trabalho (duty cycle), foram defnidos os mesmos conjuntos. As Figuras 7, 8 e 9 exibem as características dos conjuntos fuzzy criados.

\subsection{Inferência}

No estágio de inferência é que ocorrem as operações com conjuntos fuzzy, de acordo com as regras estabelecidas. Neste trabalho é usado o método MIN-MAX e a base de regras utilizada é exibida na Tabela 4.

\subsection{Defuzzyficação}

Uma vez obtido o conjunto fuzzy de saída através do processo de inferência no estágio de defuzzyficação é efetuada

Tabela 3. Funcionamento do algoritmo MPPT.

\begin{tabular}{ccc}
$\Delta P$ & $\Delta V$ & Ação sobre a tensão \\
\hline Positivo & Positivo & Incremento \\
Positivo & Negativo & Decremento \\
Negativo & Negativo & Incremento \\
Negativo & Positivo & Decremento \\
\hline
\end{tabular}

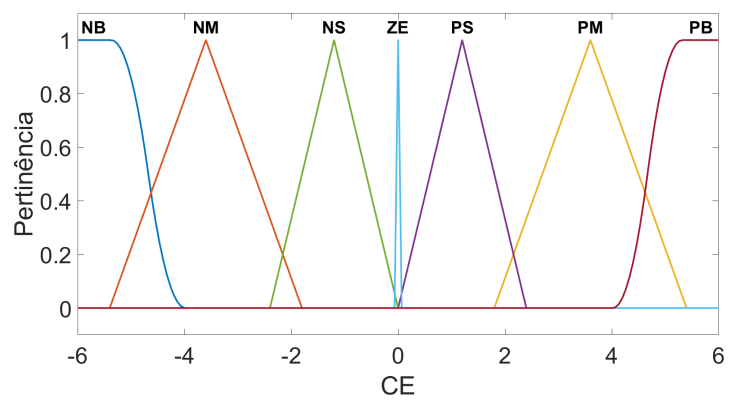

Figura 8. Conjuntos fuzzy para a variável Variação do Erro $(\mathrm{CE})$.

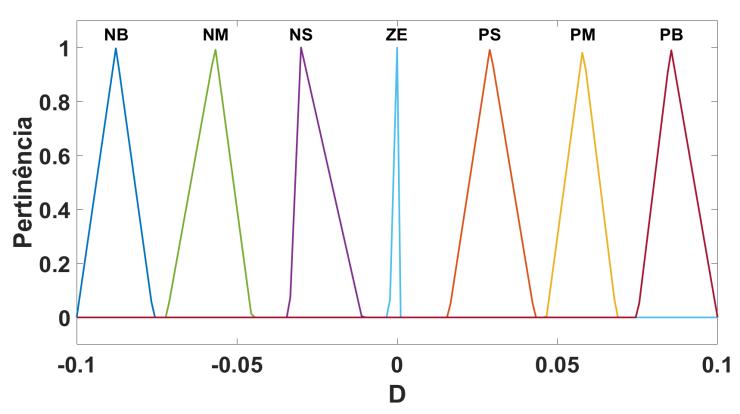

Figura 9. Conjuntos fuzzy para a variável Ciclo de Trabalho (D).

uma interpretação dessa informação. Isto se faz necessário pois, em aplicações práticas, geralmente são requeridas saídas precisas. No caso de uma sistema de controle, por exemplo, em que o controle é efetuado por um sistema de inferência fuzzy, este deve fornecer à planta dados ou sinais precisos, já que a "apresentação" de um conjunto fuzzy à entrada da planta não teria significado algum.

Foi utilizado o método centroid para se realizar a defuzzyficação e gerar a saída do sistema fuzzy. O ciclo de tabalho, resultado da inferência, é usado como parâmetro no PWM aplicado ao MOSFET do conversor CC/CC boost.

\section{RESULTADOS}

O modelo de sistema fotovoltaico foi simulado utilizando o software MATLAB/Simulink com base em um sinal de irradiação incidente, assumindo os valores de $1000 \mathrm{~W} / \mathrm{m}^{2}$ e $500 \mathrm{~W} / \mathrm{m}^{2}$, a uma temperatura inicial de $25^{\circ} \mathrm{C}$, mas que sobe para $50^{\circ} \mathrm{C}$ e os resultados do MPPT fuzzy comparados aos valores teóricos de MPPT podem ser encontrados na Tabela 5. Os resultados obtidos, podem ser avaliados se relacionados à proximidade do valor obtido pelo controlador fuzzy, comparado ao valor MPPT teórico. Esses resultados mostram que o controlador fuzzy consegue lidar

Tabela 4. Base de regras fuzzy.

\begin{tabular}{cccccccc}
$\mathbf{E} / \mathbf{C E}$ & $\mathbf{P B}$ & $\mathbf{P M}$ & $\mathbf{P S}$ & $\mathbf{Z E}$ & $\mathbf{N S}$ & $\mathbf{N M}$ & $\mathbf{N B}$ \\
\hline $\mathbf{P B}$ & PB & PM & PS & PS & NM & NM & NB \\
PM & PS & PS & PS & PS & NS & NM & NM \\
PS & PS & PS & PS & PS & NS & NS & NS \\
ZE & NS & NS & ZE & ZE & PS & NS & NS \\
NS & NS & NS & NS & NS & PS & PS & PS \\
NM & NM & NS & NS & NS & PS & PS & PM \\
NB & NB & NM & NS & NS & PS & PM & PB \\
\hline
\end{tabular}




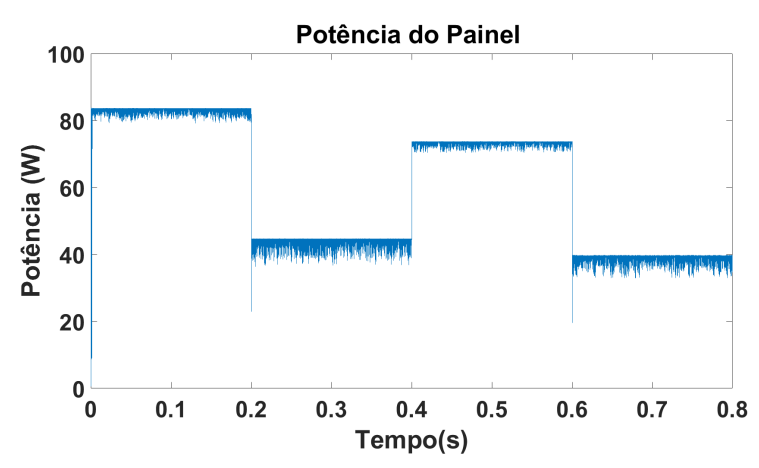

Figura 10. Potência gerada pelo painel com o controlador Fuzzy MPPT.

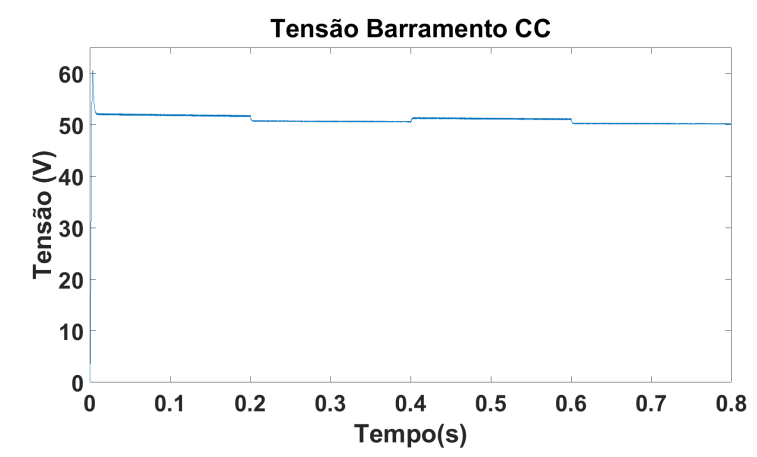

Figura 11. Tensão no Barramento CC (saída do boost) com controle por histerese.

com mudanças abruptas de entrada do sistema (Irradiação e Temperatura) e com uma média muito próxima ao valor teórico ideal. Sob a ação do controlador fuzzy MPPT, a potência gerada pelo painel é exibida na Figura 10. A tensão de saída do conversor boost pode ser obervada na Figura 11. As senóides de saída do inversor trifásico são exibidas na Figura 12. É possível perceber que há um sobresinal inicial mas que é rapidamente controlado por meio do controle por histerese do inversor.

\section{CONCLUSÃO}

O sistema proposto foi submetido à variação de radiação e temperatura e sua resposta foi observada. A precisão do algoritmo fuzzy MPPT para encontrar o ponto de máxima potência variou entre 96,4\% a 99,3\%, demonstrando ser um sistema de resposta rápida quanto às variações impostas na entrada.

Tabela 5. Valor do MPPT Teórico vs Valor do MPPT Fuzzy.

\begin{tabular}{cccc} 
Ir. $\left(\mathbf{W} / \mathbf{m}^{\mathbf{2}}\right) \mathbf{T m p} .\left({ }^{\circ} \mathbf{C}\right)$ & Teórico & Fuzzy & Média \\
\hline 1000 e 25 & $84 \mathrm{~W}$ & $83,6 \mathrm{~W} / 82,1 \mathrm{~W}$ & $82,85 \mathrm{~W}$ \\
500 e 25 & $44,74 \mathrm{~W}$ & $44,73 \mathrm{~W} / 42 \mathrm{~W}$ & $43,37 \mathrm{~W}$ \\
1000 e 50 & $73,72 \mathrm{~W}$ & $73,72 \mathrm{~W} / 72,7 \mathrm{~W}$ & $73,21 \mathrm{~W}$ \\
500 e 50 & $39,8 \mathrm{~W}$ & $39,7 \mathrm{~W} / 37,1 \mathrm{~W}$ & $38,4 \mathrm{~W}$ \\
\hline
\end{tabular}

\section{AGRADECIMENTOS}

Os autores agradecem ao Programa de Pos-Graduacão em Engenharia Elétrica da Universidade Federal de Juiz de Fora - PPEE/UFJF, à Coordenacão de Aperfeiçoamento de Pessoal de Nível Superior - CAPES pela estrutura e

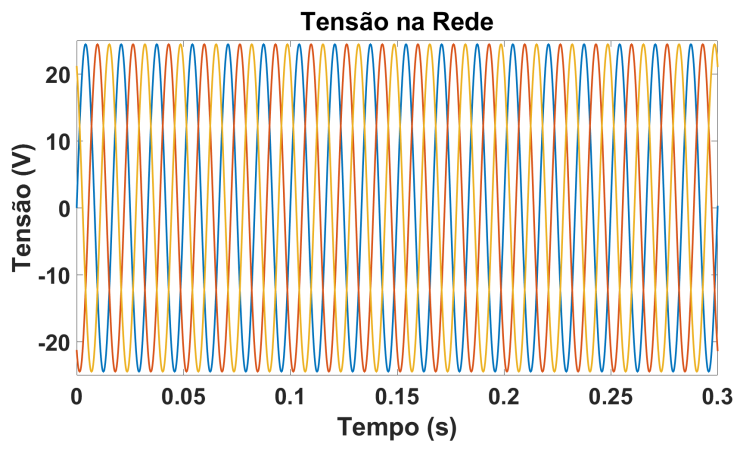

Figura 12. Sinais senoidais de saída do inversor(Tensão). apoio financeiro fornecidos para o desenvolvimento deste trabalho.

\section{REFERÊENCIAS}

Abdel-Salam, M., El-Mohandes, M.T., e Goda, M. (2018). An improved perturb-and-observe based mppt method for pv systems under varying irradiation levels. Solar Energy, 171, 547-561.

Buso, S., Fasolo, S., Malesani, L., e Mattavelli, P. (2000). A dead-beat adaptive hysteresis current control. IEEE Transactions on industry applications, 36(4), 1174-1180.

Coelho, R.F. e Martins, D.C. (2012). An optimized maximum power point tracking method based on pv surface temperature measurement. In Sustainable EnergyRecent Studies. InTech.

Farajdadian, S. e Hosseini, S.H. (2019). Design of an optimal fuzzy controller to obtain maximum power in solar power generation system. Solar Energy, 182, 161178.

Hashim, N., Salam, Z., Johari, D., e Ismail, N.F.M.N. (2018). Dc-dc boost converter design for fast and accurate mppt algorithms in stand-alone photovoltaic system. International Journal of Power Electronics and Drive Systems, 9(3), 1038-1050.

Karabacak, M. (2019). A new perturb and observe based higher order sliding mode mppt control of wind turbines eliminating the rotor inertial effect. Renewable Energy, $133,807-827$.

Khatib, T., Ghareeb, A., Tamimi, M., Jaber, M., e Jaradat, S. (2018). A new offline method for extracting iv characteristic curve for photovoltaic modules using artificial neural networks. Solar Energy, 173, 462-469.

Kumar, N., Hussain, I., Singh, B., e Panigrahi, B.K. (2018). Self-adaptive incremental conductance algorithm for swift and ripple-free maximum power harvesting from pv array. IEEE Transactions on Industrial Informatics, 14(5), 2031-2041.

Soulatiantork, P., Cristaldi, L., Faifer, M., Laurano, C., Ottoboni, R., e Toscani, S. (2018). A tool for performance evaluation of mppt algorithms for photovoltaic systems. Measurement, 128, 537-544.

Yazdani, A. e Iravani, R. (2010). Voltage-sourced converters in power systems: modeling, control, and applications. John Wiley \& Sons.

Yilmaz, U., Kircay, A., e Borekci, S. (2018). Pv system fuzzy logic mppt method and pi control as a charge controller. Renewable and Sustainable Energy Reviews, 81, 994-1001. 